\title{
Accretion and the Properties of Protobinary Systems
}

\author{
Matthew R. Bate \\ Institute of Astronomy, University of Cambridge, Madingley Road, \\ Cambridge $\mathrm{CB}_{4} 1 \mathrm{NB}$, United Kingdom
}

\begin{abstract}
We discuss the evolution of the properties of a protobinary system which forms via fragmentation within a collapsing molecular cloud core and grows to its final mass by accreting from the gaseous envelope. We predict how the mass ratio distributions and the masses of the circumstellar and circumbinary discs depend on the binary's separation, the primary's mass, and the properties of the pre-collapse molecular cloud core. We also discuss how the properties of binaries may depend on whether they were formed in isolated molecular cloud cores or in stellar clusters.
\end{abstract}

\section{Introduction}

Fragmentation of molecular cloud cores as they undergo gravitational collapse is generally considered to be the mechanism by which most binary stellar systems are formed. Fragmentation has been studied numerically for just over 20 years (e.g. Norman \& Wilson 1978; Boss \& Bodenheimer 1979; Boss 1986; Bonnell et al. 1991; Bonnell 1994; Bonnell \& Bate 1994a,b; Bate \& Burkert 1997; Burkert, Bate \& Bodenheimer 1997; Truelove et al. 1997). The overall conclusions from these studies are that fragmentation occurs relatively easily and is thus able to explain why a large fraction of stars occur in binary systems, and that fragmentation is able to produce the observed wide variety of binary properties, including large ranges in mass ratios, separations and eccentricities.

However, despite the qualitative success of the fragmentation model, it has not been possible to use it to predict quantitatively the fundamental properties of stellar systems such as the fraction of stellar systems which are binary or the properties of binary systems (e.g. the distributions of mass ratios, separations, eccentricities, and the properties of discs in pre-main-sequence systems).

There are two main reasons for this lack of predictive power. First, the results of fragmentation calculations depend sensitively on the initial conditions, which are poorly constrained. The second problem is that of accretion. When fragmentation first occurs, the binary or multiple fragments contain only a small fraction of the total mass of the original cloud (e.g. Boss 1986; Bonnell \& Bate 1994b) with the magnitude of this fraction decreasing with the binary's initial separation. To obtain the final parameters of a stellar system, a calculation must be followed until all of the original cloud material has been accumulated by one of the protostars or their discs. Unfortunately, with current computational resources, this is extremely time consuming and it is impossible to perform the 
number of calculations that would be required to predict the statistical properties of binary systems - even if we knew the distribution of initial conditions. On the other hand, if we can overcome the accretion problem, we may be able to obtain predictions of the properties of binaries and their dependence on the initial conditions, and thus use observations of the properties of binaries to better constrain the initial conditions.

\section{The Evolution of Accreting Protobinary Systems}

\subsection{The Quantitative Effects of Accretion}

The effects of the accretion of gas from an infalling gaseous envelope on the properties of a protobinary system have been studied by Artymowicz (1983), Bate (1997), and Bate \& Bonnell (1997). In particular, Bate \& Bonnell (1997) quantified the effects on the mass ratio and separation of a circular binary as functions of the specific angular momentum of the infalling gas and the binary's mass ratio.

Generally, accretion of gas with low specific angular momentum enhances the difference in stellar masses and decreases the separation of the binary, while accretion of gas with high specific angular momentum increases the binary's separation and drives the mass ratio toward unity. The effect on the mass ratio can be understood by considering an unequal-mass binary. Gas with low specific angular momentum falls toward the centre of mass of the binary which is occupied by the primary and, thus, the primary accretes most of the gas. On the other hand, gas with high specific angular momentum tends to fall in to a radius of approximately half its circularisation radius. When this radius is similar to that of the secondary's orbit around the binary's centre of mass, the secondary can accrete much more gas than the primary and so the stellar masses tend to be equalised.

Bate \& Bonnell (1997) also studied the process of disc formation in a circular binary system. They found four regimes of disc formation. First, if the binary accretes gas with low specific angular momentum, a circumstellar disc is usually formed around the primary, but no disc is formed around the secondary. The secondary does not form a disc unless the specific angular momentum of the infalling gas exceeds the specific angular momentum of the secondary around the centre of mass of the binary. The reason is that in order for gas to be captured by the secondary, by definition, it must attain the same specific orbital angular momentum as that of the secondary. If the gas has less than this value initially, it must gain some angular momentum from the secondary in order to be accreted. However, in the second regime, the specific angular momentum of the gas exceeds this value so that it has enough angular momentum to be captured and some extra to form a disc around the secondary. In the third regime, the infalling gas forms a circumbinary disc along with two circumstellar discs. The formation of a circumbinary disc occurs if the specific angular momentum of the infalling gas is similar to, or greater than, the specific angular momentum required to form a circular orbit at radius of the secondary from the binary's centre of mass. Finally, if the specific angular momentum of the gas is very large, all of the gas settles directly into a circumbinary disc and none is directly accreted from the envelope on to the circumstellar discs. 

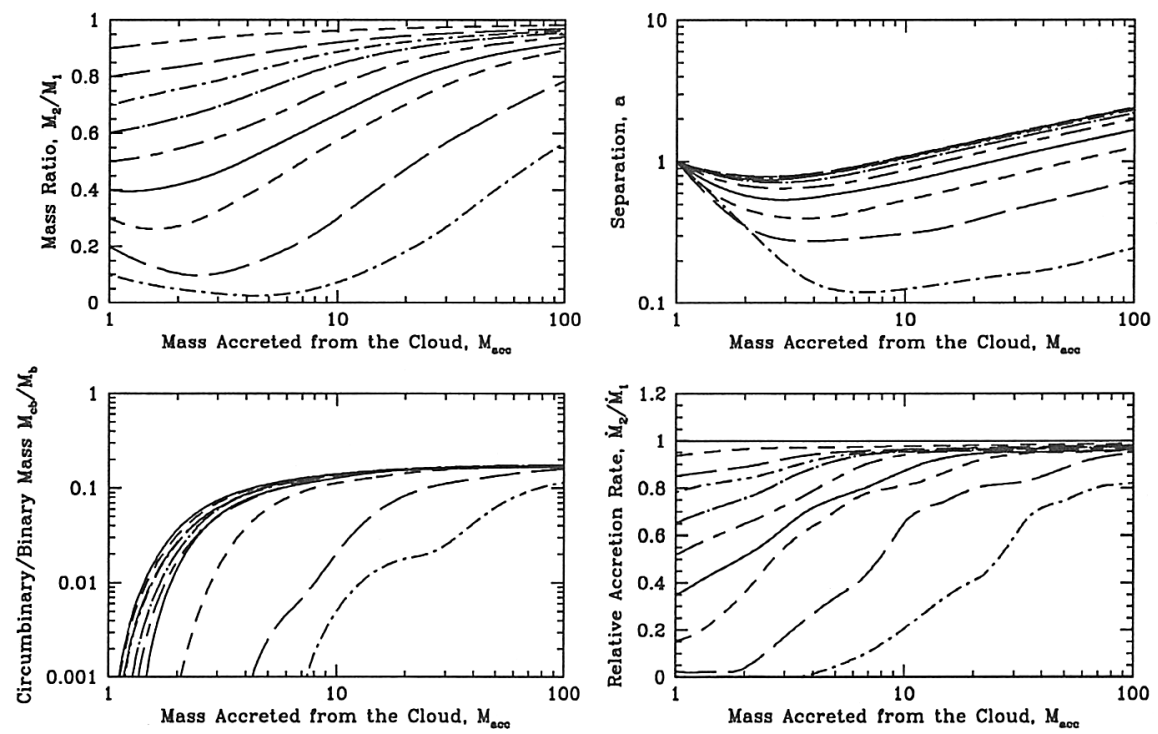

Figure 1. The panels show how a binary evolves as it accretes gas from an infalling envelope. The evolution depends on the initial mass ratio of the binary $\left(M_{2} / M_{1}\right)$ and on the properties of the pre-collapse molecular cloud core. In this case, the cores have uniform density and are in solid-body rotation before they begin to collapse dynamically. The evolution of the binary's mass ratio, separation, ratio of circumbinary disc to binary mass, and relative accretion rate on to the circumstellar discs are shown as the binary accretes up to 100 times its initial mass (see text).

\subsection{A Protobinary Evolution Code (PBE Code)}

Bate \& Bonnell (1997) determined the quantitative effects of the accretion of a small amount of gas with known specific angular momentum on to a binary with a circular orbit and a given mass ratio and separation. Using these results, Bate (2000) developed a semi-analytic method for calculating the evolution of a binary from its initial mass (immediately after its formation via fragmentation inside a collapsing molecular cloud core) to its final mass (after it has accreted all the gas from the residual envelope). This protobinary evolution (PBE) code allows one to follow how the mass ratio, separation, circumbinary-disc mass, and relative accretion rate on to the circumstellar discs evolve as the binary's mass increases. The code has been compared to the results from full hydrodynamic calculations and gives results which are at least as accurate, but are from $10^{6}$ to $10^{9}$ times faster (depending on how much mass the binary accretes).

The evolution of the properties of a binary is found to have a strong dependence on the distribution of angular momentum within the molecular cloud core before it begins to collapse dynamically (i.e. on the properties of the progenitor molecular cloud core). Figures 1, 2, and 3 show how a binary's properties evolve for three different types of progenitor molecular cloud core. The results are 

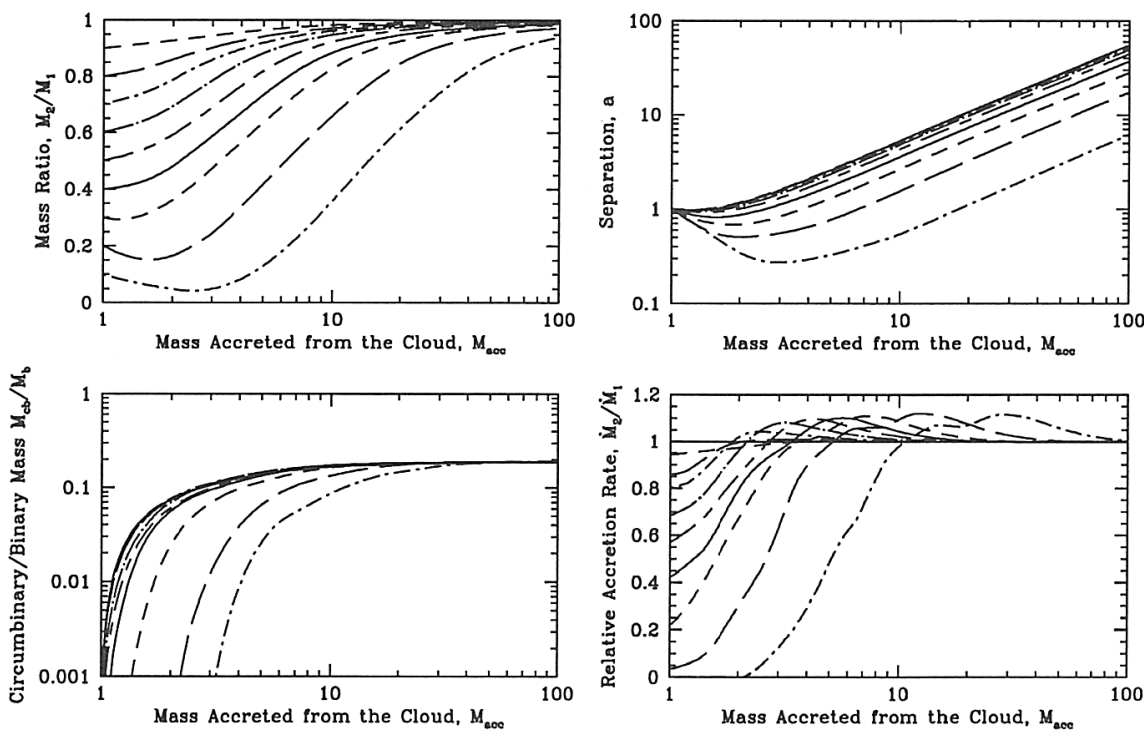

Figure 2. The same as in Figure 1, except for binaries which form in cloud cores that initially have a centrally-condensed density profile $(\rho \propto 1 / r)$ and are in solid-body rotation.
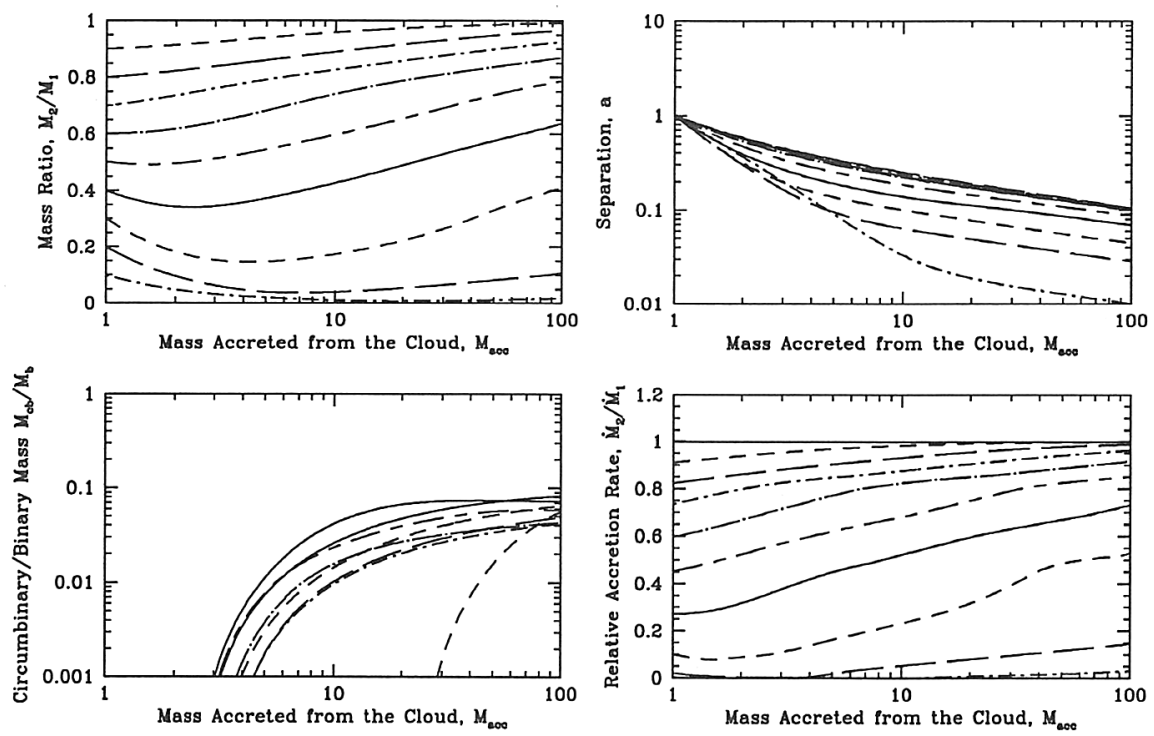

Figure 3. The same as in Figure 1, except for binaries which form in cloud cores that initially have differential rotation $(\Omega \propto 1 / r)$ and uniform density. 
given in units where the binary's initial mass and separation are unity and the rotation rate of the cloud has been set so that the gas at the centre, from which the binary was first formed, has the same total angular momentum as the orbital angular momentum of the initial binary. Other initial conditions are possible (see Bate 2000), but this choice gives the slowest possible rotation rates of the progenitor clouds, the slowest possible rate of increase of the specific angular momentum of the gas as the accretion proceeds, and, thus, the lowest possible mass ratios (for binaries with circular orbits). The free parameter in the figures is the binary's initial mass ratio. Tracks are given for protobinaries with initial mass ratios from $M_{2} / M_{1}=0.1-1.0$.

Figure 1 gives the evolution of binaries which form in the simplest possible molecular cloud core: uniform initial density in solid-body rotation. The progenitor clouds for Figure 2 also have solid-body rotation, but are centrallycondensed with density, $\rho \propto 1 / r$. The clouds in Figure 3 have uniform density, but rotate differentially with $\Omega \propto 1 / r$ such that the inner parts of the cloud rotate quicker than the outside. In terms of the distribution of angular momentum with mass, the specific angular momentum of the gas increases rapidly as mass is accreted in the centrally-condensed case and only slowly as mass is accreted in the differentially-rotating case, with the simplest case lying between the other two.

Consider the simplest case first (Figure 1). The long-term effect of accretion is to drive the binary's components toward equal masses, to increase the binary's separation, and to form a massive circumbinary disc. These effects are due to the fact that gas which falls on to the binary later in the evolution has substantially greater specific angular momentum than that early in the evolution. In the centrally-condensed case (Figure 2), the specific angular momentum of the gas increases even more rapidly as mass is accreted so that the mass ratio and separation increase much more rapidly, for the same increase in mass, than in Figure 1 and a circumbinary disc is formed earlier. In the case with differential rotation (Figure 3), the specific angular momentum of the gas increases quite slowly as the binary's mass increases and even after 100 times the initial binary's mass has been accreted it is easy to obtain small mass ratios. In this case, the binary's separation monotonically decreases and a low-mass circumbinary disc is only formed once a lot of gas has been accreted by the binary.

In order to determine the observational implications of how a binary evolves due to accretion, we must determine how much mass a typical binary accretes relative to its initial mass. Boss (1986) performed many fragmentation calculations and, for those which formed binaries, he found a linear relationship between the binary's initial mass and its separation in the isothermal collapse regime (binary separations $\gtrsim 10 \mathrm{AU}$ ). This can be understood by a simple Jeans-mass argument. For fragmentation to occur, the Jeans length at the time of fragmentation must be less than, or similar to, half the separation of the binary which is formed. However, for a fixed temperature, the Jeans mass is proportional to the Jeans length. Thus, we expect that the initial binary's mass should be roughly proportional to its separation. From Boss's results, or the simple Jeans mass argument, binaries with initial separations of $10 \mathrm{AU}$ should have initial masses of $\approx 0.01 \mathrm{M}_{\odot}$ and the initial mass of wider binaries should increase with separation (e.g. at $1000 \mathrm{AU}$, the initial mass would be $\lesssim 1 \mathrm{M}_{\odot}$ ). For close binaries (separa- 
tions $\lesssim 10 \mathrm{AU}$ ), the isothermal regime no longer applies and the binary's initial mass should be $\approx 0.01 \mathrm{M}_{\odot}$ regardless of separation. Thus, to obtain binaries with solar-mass primaries, close binaries (separations $\lesssim 10 \mathrm{AU}$ ) should have to accrete $\approx 100$ times there initial mass from the envelope, while wider binaries will have to accrete less (e.g. $100 \mathrm{AU}$ binaries may typically accrete $\approx 10$ times their initial mass). Binaries with lower final primary masses will accrete less, while binaries containing massive stars may be expected to have accreted more.

\section{Observational Implications: Isolated Star Formation}

\subsection{Mass Ratio Distributions}

Dependence on Separation From the above discussion, we find that to obtain binaries with the same final primary mass, closer systems have to accrete more, relative to the initial fragmentation mass, than wider systems. Thus, closer binaries are more likely to have mass ratios near unity (i.e. similar masses) than wider binaries since, in the long-term, accretion tends to equalise the masses. This is in good agreement with the surveys of solar-type stars. Duquennoy \& Mayor (1991) find that for binaries of all separations, the mass ratio distribution rises toward low mass ratios (i.e. unequal masses), while Mazeh et al. (1992) and Halbwachs, Mayor \& Udry (1998) find that close binaries (periods $<3000$ days, or separations $\lesssim 5 \mathrm{AU}$ ) have a uniform mass ratio distribution (i.e. they are biased toward equal masses compared to wider systems). By observing that close binaries typically have to accrete $\sim 100$ times their initial mass in order to obtain a solar-mass primary and considering the mass ratio evolutions in Figures 1 to 3, we find that it is difficult to explain the fact that there are a significant number of close binaries with low mass ratios unless there is some degree of differential rotation. Recall, however, that the evolutions are only valid for circular binaries; eccentricity allows somewhat lower mass ratios for the same increase in mass. Thus, we conclude that the observed mass ratio distributions are consistent with binaries having formed from progenitor molecular cloud cores that are in approximate solid-body rotation and with nearly-uniform density profiles (e.g. Gaussian). This is in good agreement with the observed density profiles of pre-stellar cores (Ward-Thompson et al. 1994; André, Ward-Thompson, Motte 1996; Ward-Thompson, Motte, \& André 1999). However, if the progenitor cores rotate as solid bodies it is essentially impossible to produce the observed mass-ratio distribution of close binaries if the cores are much more centrally condensed than a Gaussian-density profile (Figure 2). If differential rotation is allowed, Bate (2000) shows that cores with density profiles as steep as $\rho \propto 1 / r$ are feasible, but cores with $\rho \propto 1 / r^{2}$ still cannot reproduce the observations.

Dependence on the Binary's Total Mass If the initial mass of a protobinary depends only on its separation, and not the total mass of the cloud, then in order to obtain a more massive primary, the binary must accrete more gas from its envelope. Thus, we expect that massive binaries should have a preference for near equal masses when compared to low-mass binaries of similar separation. In fact, recent surveys seem to display the opposite result: massive stars frequently have low-mass companions. The reason for this may have to do with differences 
in the formation process of low and high-mass stars, namely that high-mass stars are preferentially formed in clusters. This will be discussed in Section 4 .

Brown Dwarf Companions Since the mass ratios of binaries are expected to become biased toward equal masses for closer binaries or higher-mass primaries, brown dwarf companions are most likely to be found in wide orbits around lowmass stars. For example, consider Figure 1 for progenitor molecular cloud cores with uniform rotation and density. To form a brown dwarf companion to a solartype star with a separation of $\lesssim 10 \mathrm{AU}$, the primary would have to accrete $\approx 100$ times its initial mass, while the final mass ratio of the binary must be $M_{2} / M_{1}<$ 0.1 for the companion to have the mass of a brown dwarf. Even allowing for eccentricity to delay the evolution toward equal masses, such companions are predicted to be extremely unlikely. This result is in good agreement with the results from radial velocity searches for planets around solar-type stars. From $\approx 600$ target stars only one brown dwarf (Zucker \& Mazeh 2000) and four candidates have been found with separations $\lesssim 2 \mathrm{AU}$, and it is possible that the four candidates are in fact stellar companions with nearly pole-on orbits (Halbwachs et al. 2000).

\subsection{Circumstellar Discs}

Figures 1 to 3 show that, regardless of the type of molecular cloud core and the initial mass ratio of the protobinary, the accretion rate from the envelope on to the secondary or its circumstellar disc is never significantly greater than that on to the primary or its disc, and is usually much lower. Thus, we predict that the primary should have a circumstellar disc which is more massive or similar to that of the secondary. The only case where a circumsecondary disc may become significantly more massive than the primary's disc is where the gaseous envelope has effectively been exhausted and the accretion on to the circumstellar discs is primarily from a circumbinary disc. In this case, because the specific angular momentum of the gas in the circumbinary disc is large and its radial velocity is low, it will be preferentially be captured by the secondary.

\subsection{Circumbinary Discs}

Just as the long-term effect of accretion on the mass ratio is to produce equalmass components, the more gas that is accreted, the more likely it is that a circumbinary disc is formed. Therefore, using the same arguments that were made for the mass ratio distributions, circumbinary discs are more likely around closer binaries or those with a greater primary mass. The dependence on separation is in good agreement with observations. Jensen, Mathieu \& Fuller (1996) found that while many close binaries (separations less than a few AU) have emission which is presumably associated with circumbinary discs, only one of their binaries with a large separation had circumbinary emission (GG Tau). Also, Dutrey et al. (1996) performed an imaging survey of 18 multiple systems that could resolve circumbinary discs with radii of $z 100 \mathrm{AU}$, but found only one circumbinary disc around UY Aur. As with the mass ratio distributions, predicting the separation where the transition from frequent to infrequent circumbinary discs should occur depends on the properties of the progenitor molecular cloud core. For binaries with solar-type primaries, Figure 2 suggests that all binaries with 

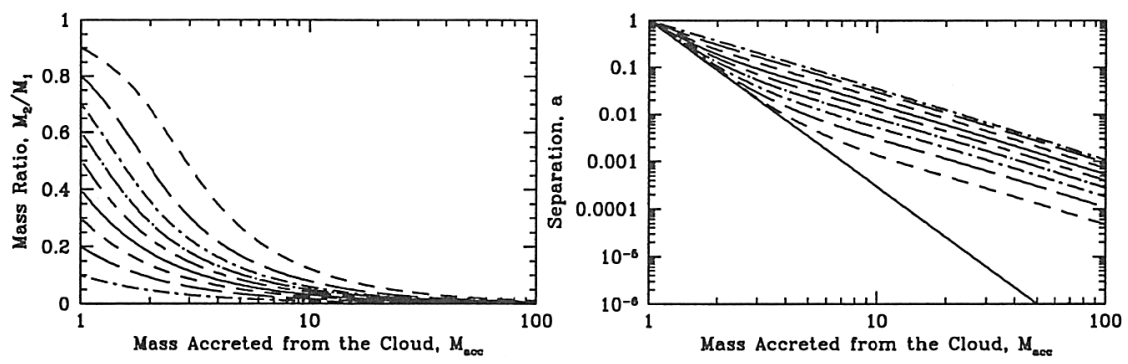

Figure 4. The same as in Figure 1, except for binaries which accrete gas with no specific angular momentum. In these cases, no circumbinary disc is formed.

separations $\lesssim 100$ AU should have circumbinary discs if the pre-collapse cores where centrally-condensed and had uniform rotation, which is not in agreement with observations. On the other hand, if cores have near uniform density profiles, most binaries with separations $\lesssim 10$ AU should have circumbinary discs, especially when allowing for eccentric systems or some differential rotation. Thus, as with the mass ratio distributions, comparison with observations favours only mildly centrally-condensed progenitor cloud cores.

\section{Observational Implications: Clustered Star Formation}

Thus far, we have assumed that all binaries form in isolated molecular cloud cores. However, many, perhaps most, stars form in dense clusters where they are expected to interact with one another on a similar time-scale to that on which they accrete the bulk of their mass. This is especially important for young intermediate and high-mass stars which are preferentially found in clusters. Bonnell et al. (1997) considered a model for the formation of a stellar cluster where a molecular cloud fragments into many protostars which subsequently move around in the residual gas accreting competitively. This leads naturally to a spectrum of masses which may be related to the initial mass function, and to the most massive stars being formed near the centre of the cluster where the potential well is deepest and the gas is densest.

In this model, the motion of the stars is generally uncorrelated with that of the gas. Thus, in contrast to the model which was discussed above where the accretion comes from a rotating molecular cloud core and, therefore, the specific angular momentum of the infalling gas increases as the mass of the binary increases, the gas accreted by a binary in a young cluster would be expected to have very little specific angular momentum throughout the entire accretion phase. The evolution of a binary which accretes gas without any angular momentum is shown in Figure 4. The stellar masses rapidly become more unequal and there is no circumbinary disc formed. In reality, of course, the gas accreted by a binary in the environment of a cluster is expected to have some angular momentum and, thus, its evolution might lie somewhere between those portrayed in Figures 3 and 4. 


\subsection{Mass Ratio Distributions}

If such a model for cluster formation is correct then, the trend of higher mass ratios (i.e. more-equal masses) with smaller separations should still be apparent because the specific angular momentum of the infalling gas, relative to the binary, will still be greater for binaries of smaller separation.

However, in comparison to isolated star formation, the trend of higher mass ratios for binaries with more massive primaries should be significantly altered and is likely to be reversed. Due to the accretion of gas with low specific angular momentum, binaries with more massive primaries (and which have therefore accreted more gas relative to their initial mass) may be expected to have mass ratio distributions which rise toward unequal masses instead of equal masses for the isolated star formation model. This is in agreement with recent surveys of open clusters (Patience, Ghez \& Reid, in preparation) which find that the mass ratio distributions exhibit a steeper rise towards low mass ratios for higher-mass primaries than low-mass primaries.

We note that while young high-mass stars $\left(M \gtrsim 3 \mathrm{M}_{\odot}\right)$ are preferentially associated with clusters, low-mass stars are formed in isolated star-forming regions (SFRs) as well as in clusters. An obvious implication of this is that the mass ratio distributions of low-mass stars may differ between isolated and clustered SFRs. Indeed, it has been observed that there is a much higher fraction of binaries with components of nearly equal brightness in the Taurus SFR (an isolated SFR) than in the Ophiuchus SFR where the star formation is predominantly in a small cluster (Duchêne 1999).

\subsection{Circumstellar Material}

We saw earlier that, with isolated star formation, the mass of a circumprimary disc should always be greater than, or similar to, that of the secondary's disc. This still applies for clustered star formation, except that the primary's disc should dominate that of the secondary even more strongly. Due to the low specific angular momentum of the gas accreted by binaries in clusters, however, we would only expect to find circumbinary discs around very close binaries.

\section{Conclusions}

Until now, except in the simplest case of scale-free fragmentation (Clarke 1996), it has been impossible to predict the statistical properties of binary systems which are formed by fragmentation. However, by studying the evolution of a binary as it accretes gas from a surrounding envelope, we can obtain predictions for the mass ratios of binaries and the distribution of circumstellar material and determine how a binary's properties depend on the properties of the molecular cloud core before it began to collapse. Hopefully, this will stimulate further interaction and comparison between observational and theoretical studies of binary star formation so that we are able to refine and/or eliminate models.

There is still much observational effort required for us to determine the statistical properties of binaries with enough accuracy to provide strong constraints on theory. Some of the main areas for observational work include: determining the dependence of mass ratios on separation and primary mass; determining 
the dependence of circumbinary-disc masses on separation and primary mass; and looking for differences in the properties of binaries with low-mass primaries $\left(\varsigma 1 \mathrm{M}_{\odot}\right)$ in regions of distributed star formation compared to clusters. On the theoretical side, the evolution of accreting binaries is currently limited to circular binaries. The evolution of eccentric binaries is currently being investigated and may lead to predictions for the eccentricity distributions of binary stars.

\section{References}

André, P., Ward-Thompson, D., Motte, F. 1996, A\&A, 314, 625

Artymowicz, P. 1983, Acta Astronomica, 33, 223

Bate, M. R. 1997, MNRAS, 285, 16

Bate, M. R. 2000, MNRAS, 314, 33

Bate, M. R., Bonnell, I. A. 1997, MNRAS, 285, 33

Bate, M. R., Burkert, A. 1997, MNRAS, 288, 1060

Bonnell, I. A. 1994, MNRAS, 269, 837

Bonnell, I. A., Bate, M. R. 1994a, MNRAS, 269, L45

Bonnell, I. A., Bate, M. R. 1994b, MNRAS, 271, 999

Bonnell, I. A., Bate, M. R., Clarke, C. J., Pringle, J. E. 1997, MNRAS, 285, 201

Bonnell, I., Martel, H., Bastien, P., Arcoragi, J. -P., Benz, W. 1991, ApJ, 377, 553

Boss, A. P. 1986, ApJS, 62, 519

Boss, A. P., Bodenheimer, P. 1979, ApJ, 234, 289

Burkert, A., Bate, M. R., Bodenheimer, P. 1997, MNRAS, 289, 497

Clarke, C. J. 1996, MNRAS, 283, 353

Duchêne, G. 1999, A\&A, 341, 547

Duquennoy, A., Mayor, M. 1991, A\&A, 248, 485

Dutrey, A., Guilloteau, S., Duvert, G., Prato, L., Simon, M., Schuster, K., Menard, F. 1996, A\&A, 309, 493

Halbwachs, J. L., Arenou, F., Mayor, M., Udry, S., Queloz, D. 2000, A\&A, 355, 581

Halbwachs, J. L., Mayor, M., Udry, S. 1998, in ASP Conf. Ser. Vol. 134, Brown Dwarfs and Extrasolar Planets, ed. R. Rebolo, E. L. Martin \& M. R. Zapatero Osorio (Provo: Brigham Young University), 308

Jensen, E. L. N., Mathieu, R. D., Fuller, G. A. 1996, ApJ, 458, 312

Mazeh, T., Goldberg, D., Duquennoy, A., Mayor, M. 1992, ApJ, 401, 265

Norman, M. L., Wilson, J. R. 1978, ApJ, 224, 497

Truelove, J. K., Klein, R. I., McKee, C. F., Holliman, J. H., Howell, L. H., Greenough, J. A. 1997, ApJ, 489, L179

Ward-Thompson, D., Motte, F., André, P. 1999, MNRAS, 305, 143

Ward-Thompson, D., Scott, P. F., Hills, R. E., André, P. 1994, MNRAS, 268, 276

Zucker, S., Mazeh, T. 2000, ApJ, 531, L67 